$$
\begin{aligned}
& \text { شناسابى مولكولى كو كسيلا بورنتى در كنههاى جدا شده از بزهاى شهرستان مشكين } \\
& \text { شهرو، استان اروبيل، إبران }
\end{aligned}
$$

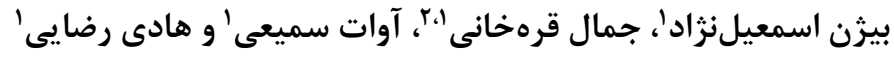

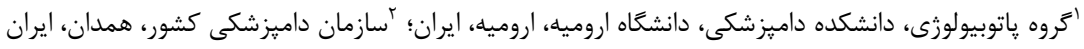

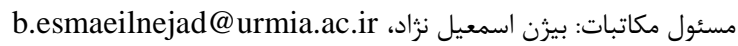

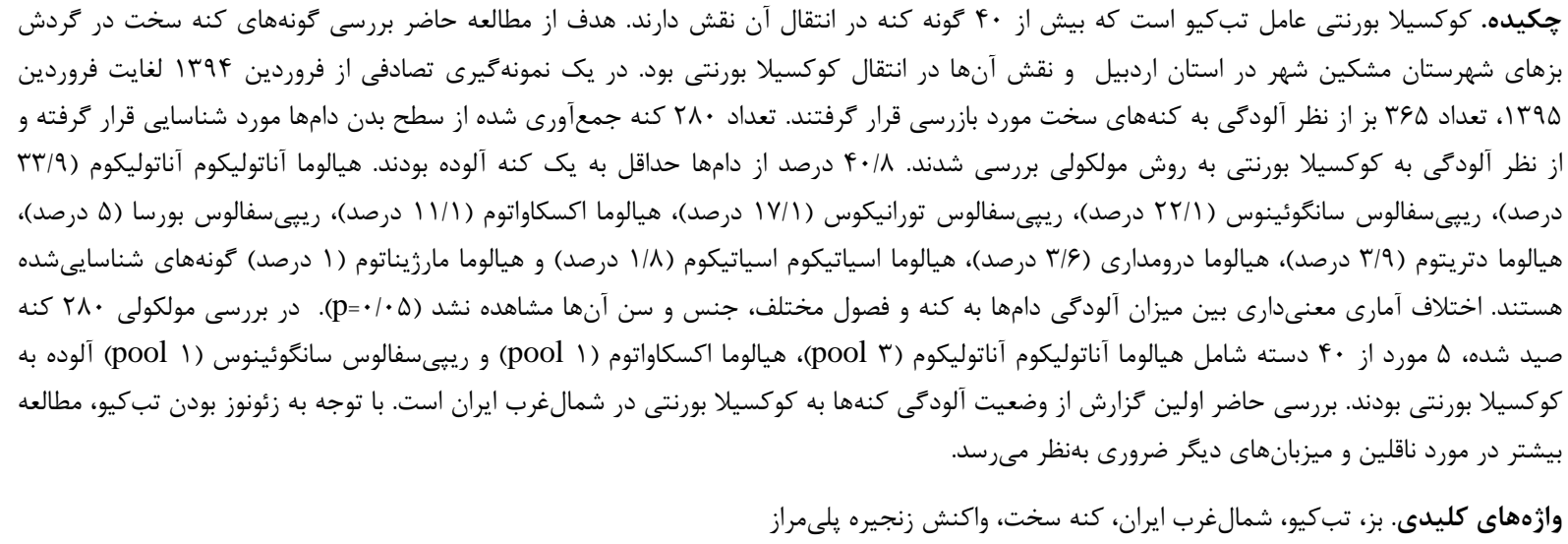

\title{
Molecular detection of Coxiella burnetii in ticks isolated from goats of Meshkin-Shahr County, Ardabil Province, Iran
}

\author{
Bijan Esmaeilnejad ${ }^{1}$, Jamal Gharekhani ${ }^{1,2}$, Awat Samiei ${ }^{1}$ \& Hadi Rezaei ${ }^{1}$ \\ ${ }^{1}$ Department of Pathobiology, Faculty of Veterinary Medicine, Urmia University, Urmia, Iran; ${ }^{2}$ Iranian Veterinary \\ Organization, Hamedan, Iran \\ Correspondent author: Bijan Esmaeilnejad, b.esmaeilnejad@urmia.ac.ir
}

\begin{abstract}
Q fever is caused by Coxiella burnetii and is transmitted by more than 40 tick species. This study aimed to identify the tick species in goats of Meshkin-Shahr, Ardabil Province, Iran, and their role in the transmission of $C$. burnetii. A number of 365 goats were inspected for the infestation of hard ticks in a randomized sampling design, during a 1-year period from April 2013 to April 2014. A total number of 280 tick specimens were collected and identified and were then examined by molecular methods for the presence of C. burnetii infection. $40.8 \%$ of the infected animals had at least one species of tick. The goats which were studied were found to be infested by the following tick species: Hyalomma anatolicum anatolicum (33.9\%), Rhipicephalus sanguineus (22.1\%), Rhipicephalus turanicus (17.1\%), Hyalomma excavatum (11.1\%), Rhipicephalus bursa (5\%), Hyalomma detritum (3.9\%), Hyalomma dromedarii (3.6\%), Hyalomma asiaticum asiaticum (1.8\%), and Hyalomma marginatum (1\%). There were no significant differences between the infestation rate in different seasons, genders and ages of the animals which were studied ( $\mathrm{p}=0.05$ ). The molecular assay of 280 tick specimens revealed the presence of $C$. burnetii infection in 5 out of 40 pool samples (6-8 tick individuals of the same species in each pool), including H. anatolicum anatolicum ( 3 pools), $H$. excavatum (1 pool) and $R$. sanguineus (1 pool). The present study, therefore, reports the rate of $C$. burnetii infection transmitted by hard ticks in North-West of Iran for the first time. According to the zoonotic aspect of Q fever, further studies on the carriers as well as other hosts of the infection were found to be necessary.
\end{abstract}

Key words. goat, hard tick, north-east Iran, PCR, Q fever 
است (Nourollahi-Fard \& Khalili, 2011). در يك مطالعهـ

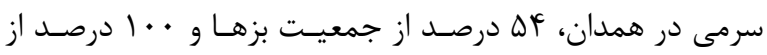

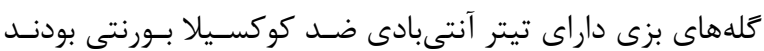
TV/T Edalati Shokat et al., 2015) درصد از بزهاى ايران از نظر سرمى مثبت تزارش شدند ( Asadi et al., 201

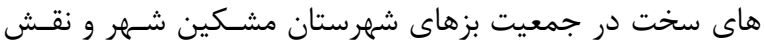

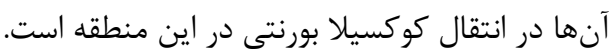

\section{مواد و روشها}

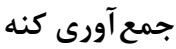

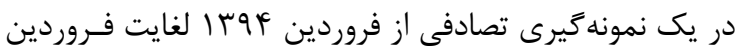

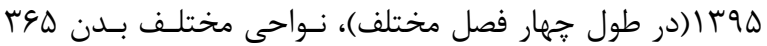

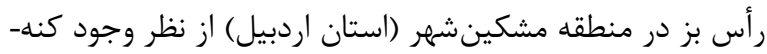

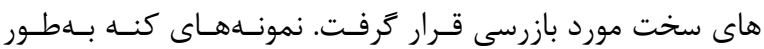

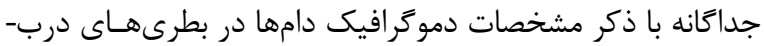

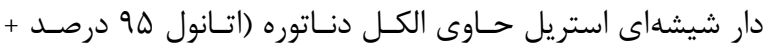

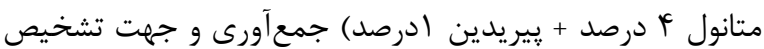

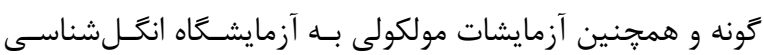

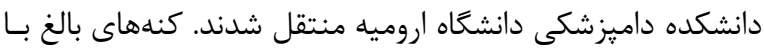

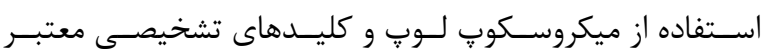

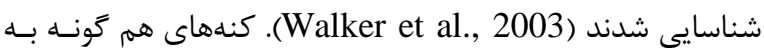
Fool) (pol) تقسيم شدند (هر دسته شامل ^-4 كنه). سويه استاندارد كو كسيلا بورنتى

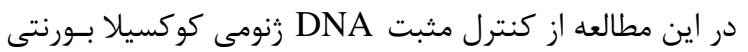

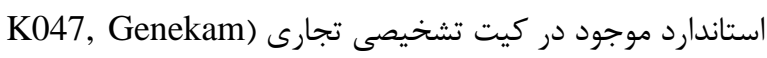
Biotechnology AG, Germany

\section{DNA استخراج}

براى استخراج DNA كوكسيلا بورنتى، ابتدا كنـهـهـا از اتـانول

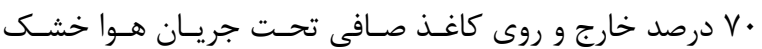

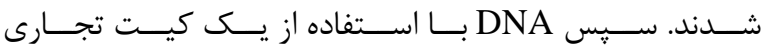
(DNA Extraction Kit, MBST, Iran)

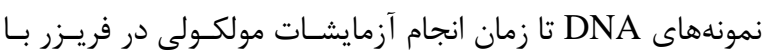
دماى · ·- درجه سانتى كراد نكمهدارى شدند.

Nested Trans- PCR اين روش به دليل استفاده از يرايمرهاى زن IS1111 داراى

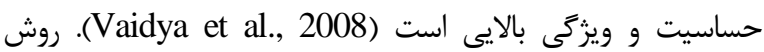

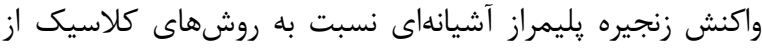

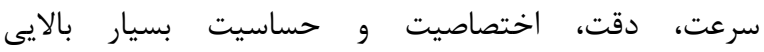

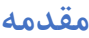

تبكيو (Q fever) يكى از بيمارىهاى مشترك بين انسـان و دام

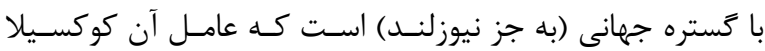

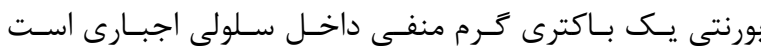
(Marrie, 1995; Khalili et al., 2011)

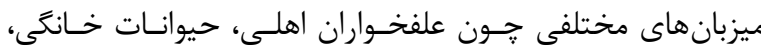

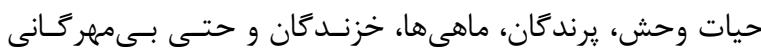

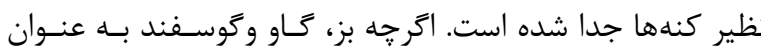

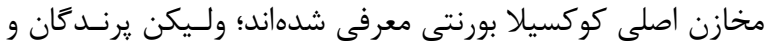

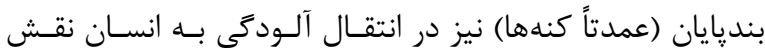
برجستهاى دارنـد (Nourollahi-Fard \& Khalili, 2011). كنههـا در انتقـال طيـف وسـيعى از عوامـل بيمـارىزا (بـاكترى،

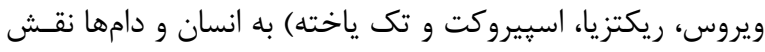

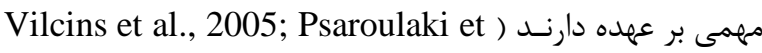

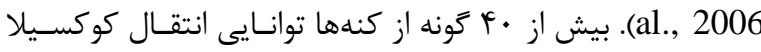

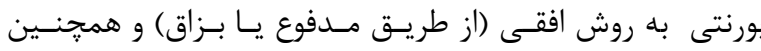
عمودى (از طريق تخمم) را دارند (Kazar, 2005).

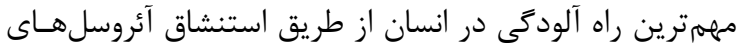

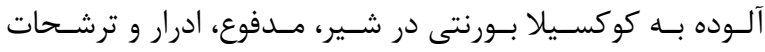

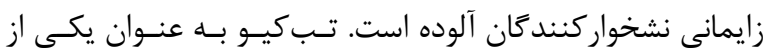

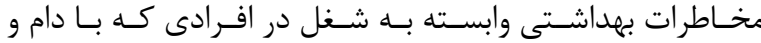

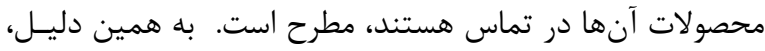

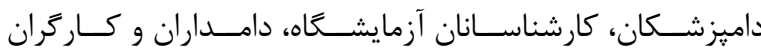

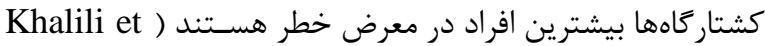

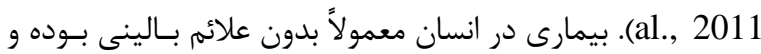

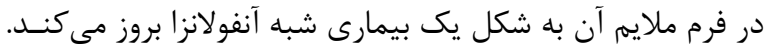

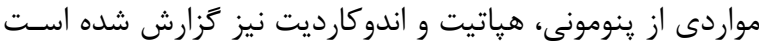

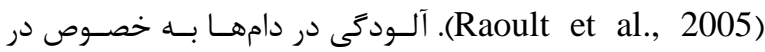

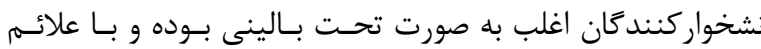

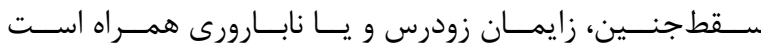

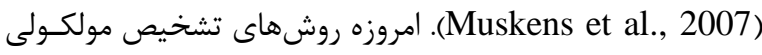

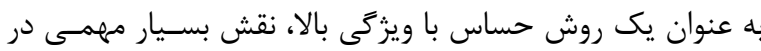

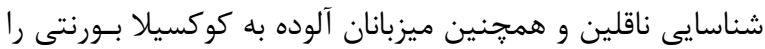
دارند (Capin et al., 2013). كزارشات متعددى از وقوع تبكيو در مناطق مختلف جهان بـهـ خصوص كشورهاى همسايه ايران (عراق، افغانسـتان و تركيـه) در دسترس است (ئ) Leung-Shea \& Danaher, 2006; Bailey et

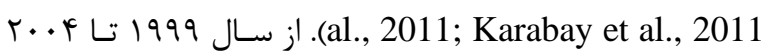
سه طغيان بيمارى تبكيو از جمعيت بزها در جهان گزارش شـده 
جدول ا- مشخصات برايمرهاى مورد استفاده در تشخيص كو كسيلا بورنتى.

Table 1. The primers used in the diagnosis of C. burnetii.

\begin{tabular}{|c|c|c|c|}
\hline طول قطعات (bp) & 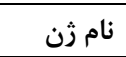 & توالى يرايمرها & نام يرايمرها \\
\hline \multirow[t]{2}{*}{687} & IS1111 & $5^{\prime}$ TATGTATCCACCGTAGCCAGTC 3' & Trans1 \\
\hline & & $5^{\prime}$ CCCAACAACACCTCCTTATTC $3^{\prime}$ & Trans2 \\
\hline \multirow[t]{2}{*}{687} & IS1111 & 5' TATGTATCCACCGTAGCCAGTC 3' & Trans 1 \\
\hline & & $5^{\prime}$ CCCAACAACACCTCCTTATTC $3^{\prime}$ & Trans2 \\
\hline \multirow[t]{2}{*}{203} & & 5’ GAGCGAACCATTGGTATCG 3' & $261 \mathrm{~F}$ \\
\hline & & $5^{\prime}$ CTTTAACAGCGCTTGAACGT 3' & $463 \mathrm{R}$ \\
\hline
\end{tabular}

محاسبه P-value با سطح معنىدار كوجكتر از هـ/• استفاده شد. همجنين از ركرسيون خطى براى تعيين اثر شاخصهاى

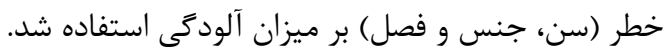

نتايج

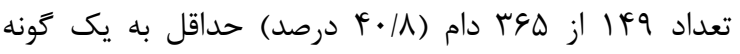

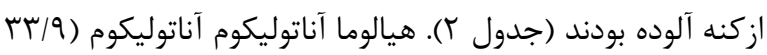

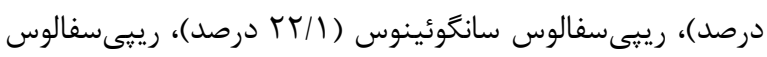

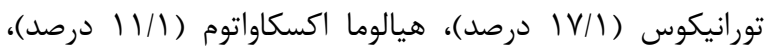

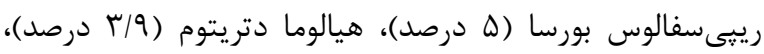

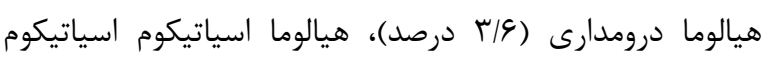

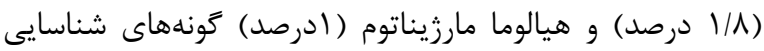

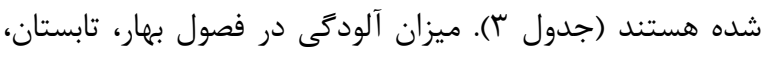

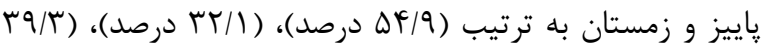

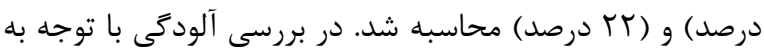

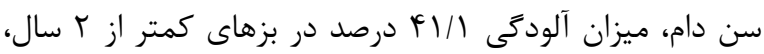

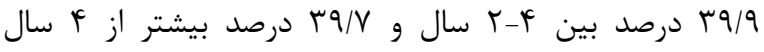

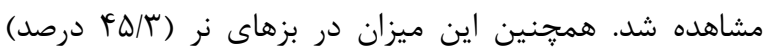

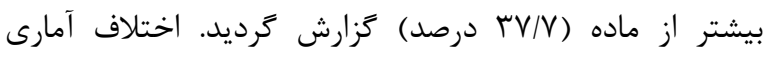

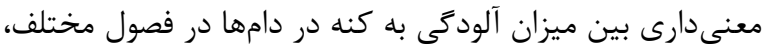

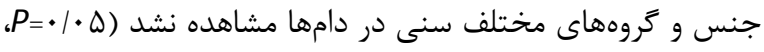

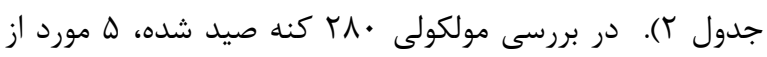
أ.

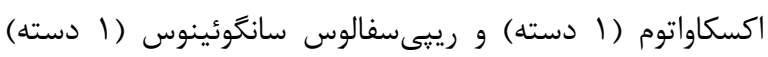
آلوده به كوكسيلا بورنتى بودند (شكل () (1).

بحث كنهها از شايعترين كونههاى بنديايان هستند كه توانايى آلودهكردن كونهاى مختلف حيوانات اهلى و وحشى و همجنين انسان را را دارند.

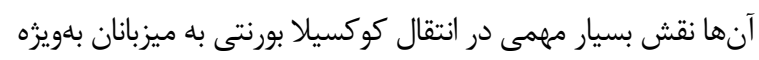

برخوردار است؛ به همين دليل از اين روش براى تشخيص كوكسيلا

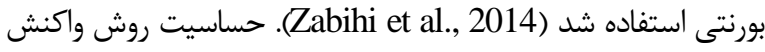

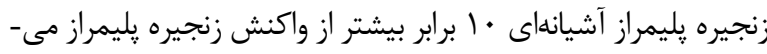

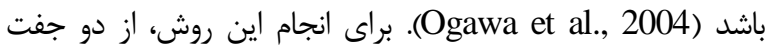

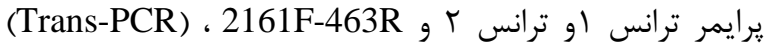
براساس قطعات شبهترانس يوزيون ترانس ترارى (Trans-PCR) استفاده شد (جدول () (Parisi et al., 2006).

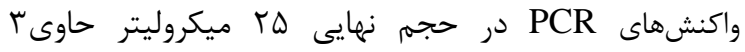

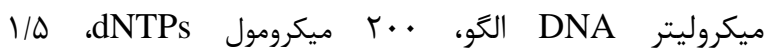

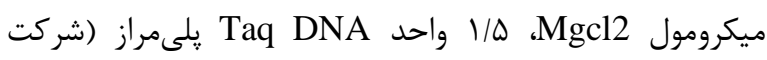

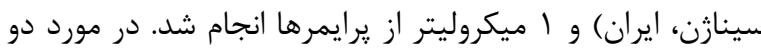

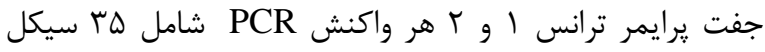

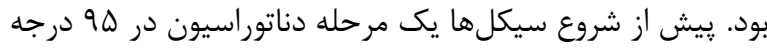

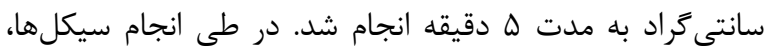

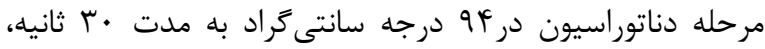

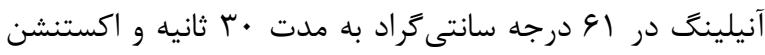

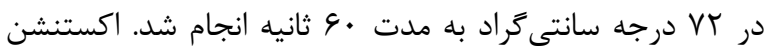

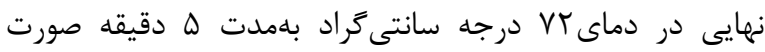

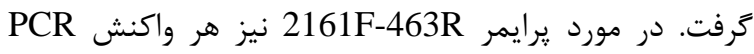

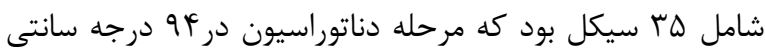

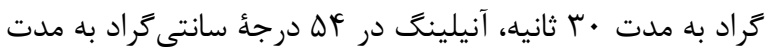

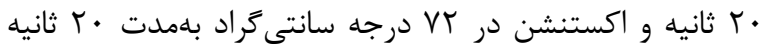
انجام شد (Nourollahi-Fard \& Khalili, 2011). ֶّ از

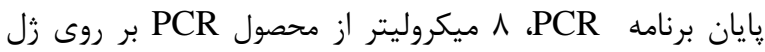

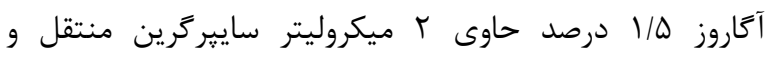

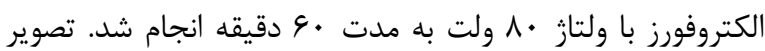

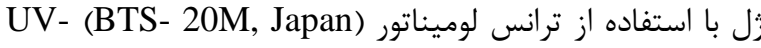
تجلم Transilluminator تحليل دادهها

College ) Stata, version 11.2 دادها با استفاده ادهان تحليل شدند. از آزمون مربع كاى جهت (Station, TX, USA 


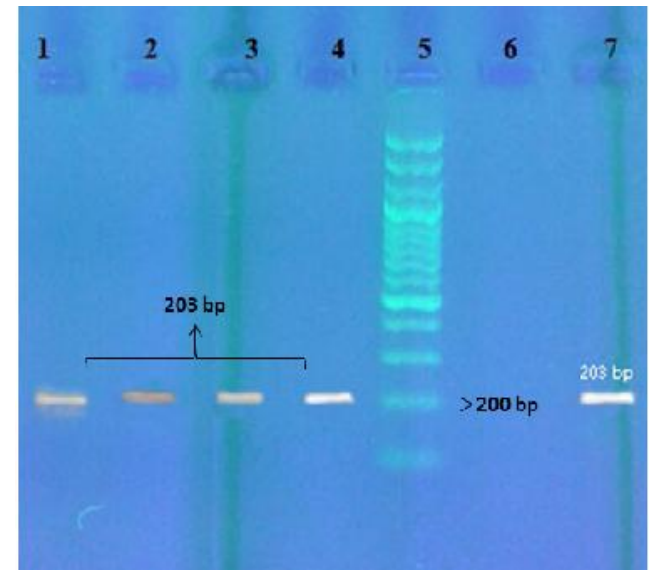

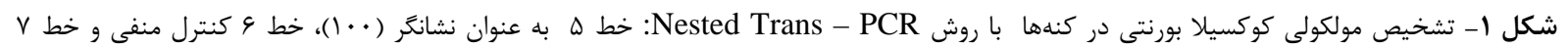

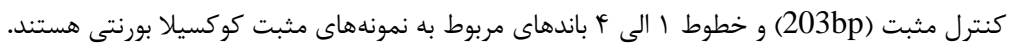

Fig. 1. Molecular identification of Coxiella burnetii in ticks by means of nested trans- PCR method: Line 5 (marker 100), line 6 (negative control), Line 7 (positive control) and line 1-4 (positive samples of C. burnetii).

$$
\text { جدول r- فراوانى آلودگى بزها به كنهاى سخت براساس فصل، جنس و سن دام در مشكين شهر. }
$$

Table 2. Infestation rate of the goats of Meshkin-Shahr with hard ticks, on the basis of the season of sampling and the sex and the age of animals studied.

\begin{tabular}{|c|c|c|c|c|}
\hline$P$-value & (درصد) تعداد دام آلوده & تعداد دام نمونه گيرىشده & & 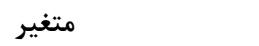 \\
\hline \multirow[t]{4}{*}{$>\cdot / \cdot \Delta$} & $V \wedge(\Delta F / q)$ & IFT & 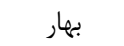 & \multirow[t]{4}{*}{ فصل } \\
\hline & rG(Tr/l) & 111 & تابستان & \\
\hline & $r F(r q / \Gamma)$ & 91 & ياييز & \\
\hline & W(Tr) & $\Delta \cdot$ & مستان & \\
\hline \multirow[t]{3}{*}{$>\cdot 1 \cdot 0$} & GQ(FT/I) & 194 & كمتر از r & \multirow[t]{3}{*}{ كروه هاى سنى (سال) } \\
\hline & $\Delta \Delta(\Upsilon q / ৭)$ & Irk & $r-r$ & \\
\hline & $r \Delta(r q / V)$ & qr & بيشتر از f & \\
\hline \multirow[t]{3}{*}{$>\cdot 1 \cdot 0$} & $G \wedge(\mathcal{F} Q / \Gamma)$ & 10 & نر & \multirow[t]{2}{*}{ جنس } \\
\hline & $\Lambda I(\mathrm{YV} / \mathrm{V})$ & rid & 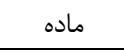 & \\
\hline & $\mid f q(\uparrow \cdot / \Lambda)$ & $r \varphi \Delta$ & & جمع \\
\hline
\end{tabular}

جدول r- فراوانى و تنوع گونهاى كنهاى سخت جدا شده از بزهاى مشكين شهر.

Table 3. Prevalence and diversity of hard ticks collected from the goats of Meshkin-Shahr.

\begin{tabular}{|c|c|c|}
\hline فراوانى & تعداد جمع آورى شده & كونه كاى كنه \\
\hline 童 & $9 \Delta$ & هيالوما اناتوليكوم اناتوليكوم \\
\hline$r r / l$ & Gr & ريبيىسفالوس سانكوئينوس \\
\hline$|V /|$ & ¿A & رييىسفالوس تورانيكوس \\
\hline $11 / 1$ & r & هيالوما اكسكاواتوم \\
\hline$\Delta$ & if & رييى سفالوس بورسا \\
\hline$r / 9$ & 11 & هيالوما دتريتوم \\
\hline$r / 9$ & 1. & هيالوما درومدارى \\
\hline $1 / \Lambda$ & $\Delta$ & هيالوما اسياتيكوم اسياتيكوم \\
\hline 1 & f & هيالوما مارزيناتوم \\
\hline $1 \cdots$ & rA. & جمع \\
\hline
\end{tabular}


معكوسى با دماى محيط دارد. بيشترين تعداد كنه در دماى • ·؟-

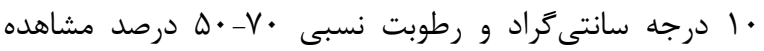

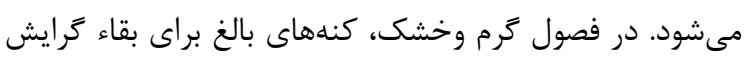
به سطح زمين و استقرار در سطوح خاك دارند. اين زمان بهترين

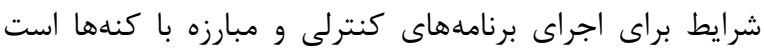
(Noaman et al., 2008)

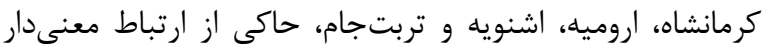

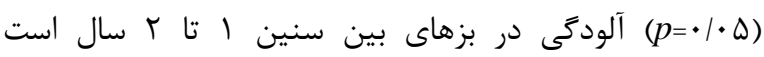

Yakhchali \& Hajihasanzadehzarza, 2004; ) Yakhchali \& Hosseine, 2006; Yakhchali \& Azizi, 2007; Yakhchali \& Ranjbargarmabolia, 2008; Sohrabi et al., 2013

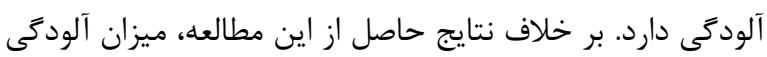

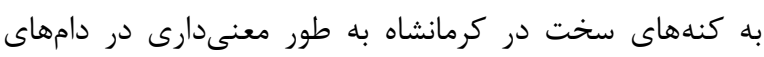
ماده بيشتر از نرها كزارش شده است (Sohrabi et al., 2013).

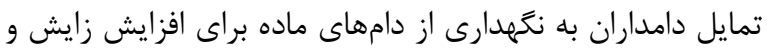

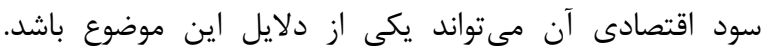

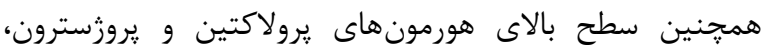

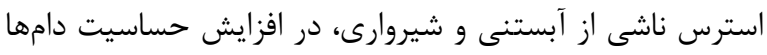

$$
\text { به آلودَى هاى انغَلى بسيار موثر است. }
$$

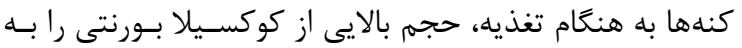

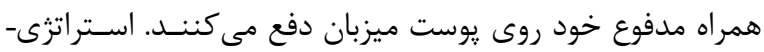

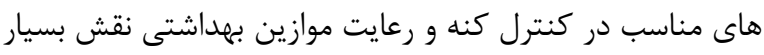

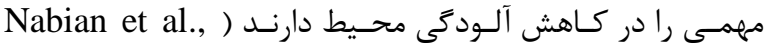

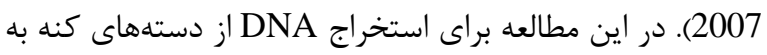

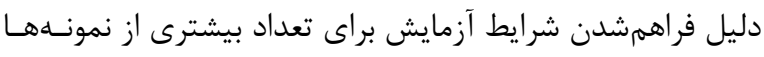

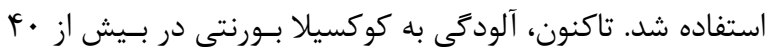

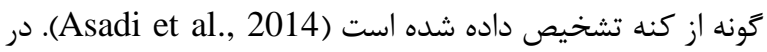

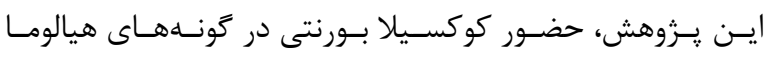

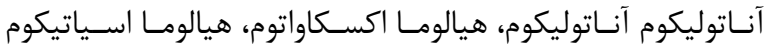

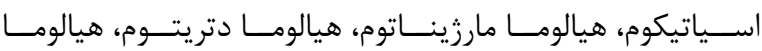

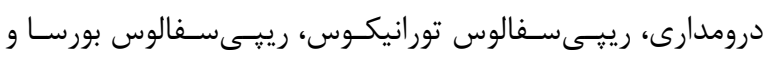

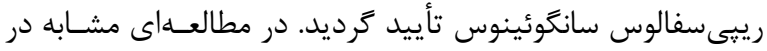

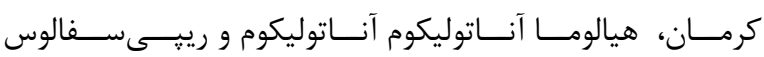

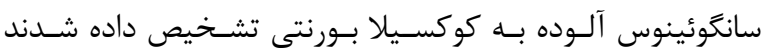
Nourollahi-Fard \& Khalili, 2011)

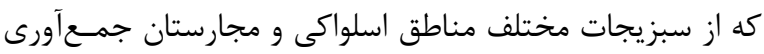

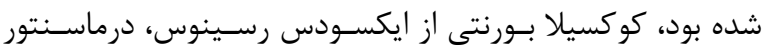

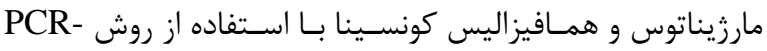

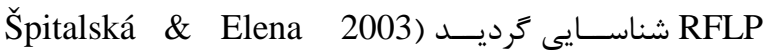

Angelakis \& Raoult, ) جوندكان و يرندكان وحشى را دارند

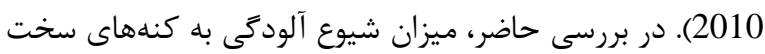

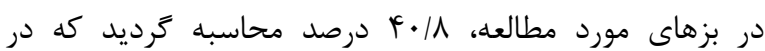

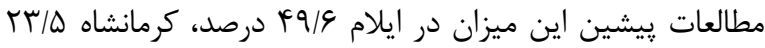

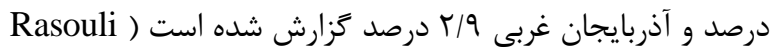
(et al., 2010; Sohrabi et al., 2013

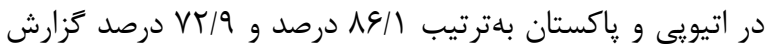
شده است (Werede \& Afera, 2014; Sajid et al., 2009). عمده تفاوتها در نتايج به دليل توزيع جغرافيايى، شرايط اقليمى و سيستمهاى مديريت در يرورش دام است (Sajid et al., 2009).

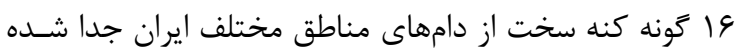

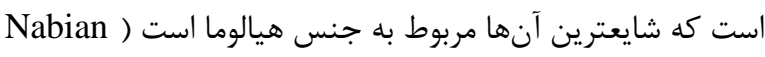
al., 2007; Nabian \& Rahbari, 2008

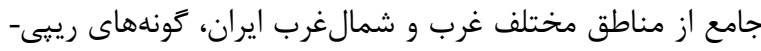

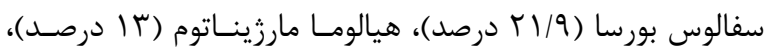

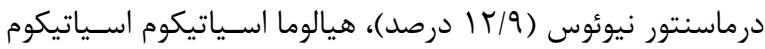

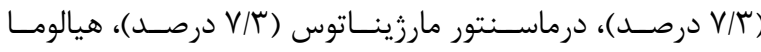

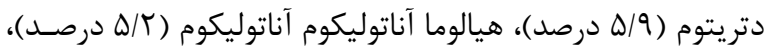

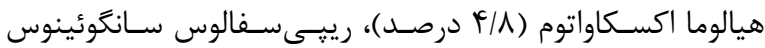

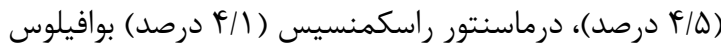

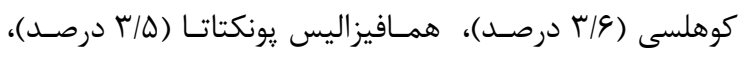

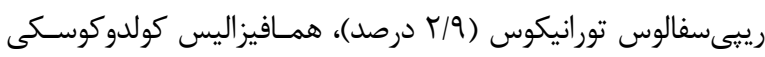

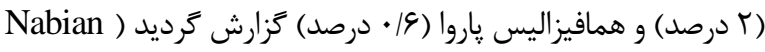

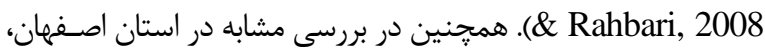

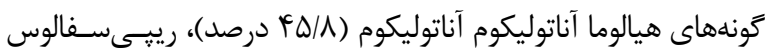

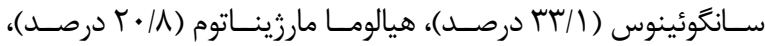

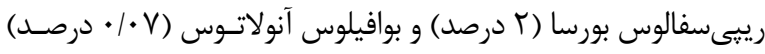

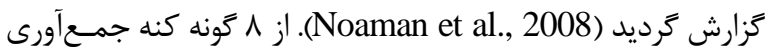

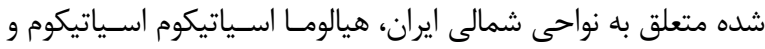

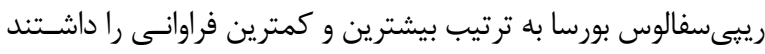
.Keyhani et al., 2013) در مطالعه حاضر هيجَّونه ارتباط آمارى معنى دارى بين ميزان

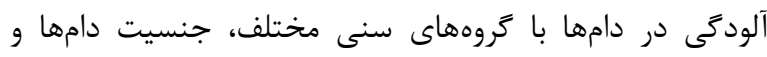

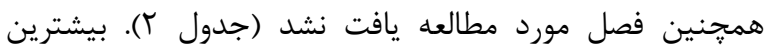
ميزان آلودگى در جمعيت بزهاى شهرستان مياندوآب در دران

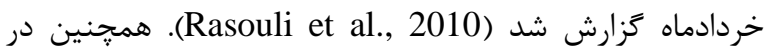

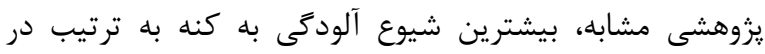

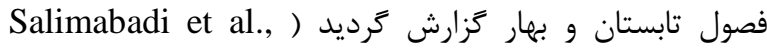

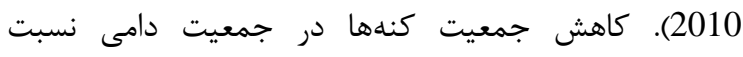

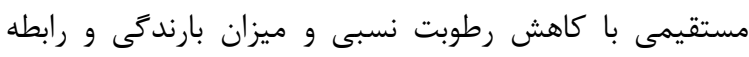




\section{REFERENCES}

Angelakis, E. \&Raoult, D. 2010. Q fever. Veterinary Microbiology 140: 297-309.

Asadi, H., Khalili M., Kafi, M., Ansari Lari, \& M. Hosseini, S.M. 2014. Risk factors of Q fever in sheep and goat flocks with history of abortion. Comparative Clinical Pathology 23: 625-630.

Bailey, M.S., Trinick, T.R., Dunbar, J.A., Hatch, R., Osborne, J.C., Brooks, T.J. \& Green, A.D. 2011. Undifferentiated febrile illnesses amongst British troops in Helmand, Afghanistan. The Journal of the Royal Army Medical Corps 157: 150-155.

Capin, G.A., Emre, Z., Canpolat, S., Vatansever, Y. \& Duzgun, A. 2013. Detection of Coxiella burnetii from ticks by Polymerase Chain Reaction and restriction fragment length polymorphism. Ankara University Veterinary Faculty Journal 60: 263-268.

Edalati Shokat, H., Abbasi Doulatshahi, E., Hajian Bidar, H., Gharekhani, J. \& rezaei, A.A. 2015. Q fever in domestic ruminants: a seroepidemiological survey in Hamedan, Iran. International Journal Current Microbiology and Applied Science 4: 1-9.

Karabay, O., Gozdas, H.T., Ozturk, G., Tuna, N. \& Utku, A.C. 2011. A Q fever case mimicking Crimean-Congo haemorrhagic fever. Indian Journal of Medical Microbiology 29: 418.

Kazar, J. 2005. Coxiella burnetii infection. Annals of the New York Academy of Sciences 1063: 105-114.

Keyhani, A., Nejati, J., Rostami, A., Hosseini, M., Davari, B. \& Mousa-Kazemi, S.H. 2013. Study on hard tick species and their infection to Enterobacteriacea in animals in Amol. Scientific Journal of Medical University of Kurdistan 17: 78-85.

Khalili, M., Rezaei, M., Akhtardanesh, B., Abiri, Z. \& Shahheidaripour, S. 2018. Detection of Coxiella burnetii (Gammaproteobacteria: Coxiellaceae) in ticks collected from infested dogs in Kerman, Southeast of Iran. Persian Journal of Acarology 7: 93100.

Khalili, M., Shahabi-Nejad, N. \& Aflatoonian, M.R. 2011. Q fever a forgotten disease in Iran. Journal of Kerman University of Medical Sciences 18: 93-97

Leung-Shea, C. \& Danaher, P.J. 2006. Q fever in members of the United States armed forces returning from Iraq. Clinical Infection Diseases 43: 77-82.

Marrie, T.J. 1995. Coxiella burnetii (Q fever) pneumonia. Clinical Infection Diseases 21: 253-264.

Muskens, J., Mars, M.H. \& Franken, P. 2007. Q fever: an overview. Tijdschr Diergeneeskd 132: 912-917.

Nabian, S. \& Rahbari, S. 2008. Occurrence of soft and hard ticks on ruminants in Zagros mountainous areas of Iran. Iranian Journal of Arthropod-Borne Diseases 2: 16-20.

Nabian, S., Rahbari, S., Shayan, P. \& Haddadzadeh, H.R. 2007. Current status of tick fauna in North of Iran. Iranian Journal of Parasitology 2: 12-17.

Noaman, V., Abdi-Goudarzi, M., Nabinejad, A.R., Heidari, M.R. \& Khalilifard, M. 2008. Identification of hard ticks of domestic ruminants in two ecological zones of Isfahan province, Iran. Pajouhesh \& Sazandegi 77: 88-95.

$$
\begin{aligned}
& \text { Kocianova, }
\end{aligned}
$$

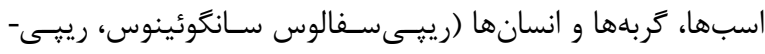

$$
\begin{aligned}
& \text { سـفالوس تورانيكـوس و درماســنتور مارزينــاتوس) بــهـ كوكسـيلا } \\
& \text { بورنتى با اسـتفاده از روش Real time PCR تاييـد شــده اسـت } \\
& \text {-Socolovschi et al., 2012) } \\
& \text { سفالوس سانگُوئينوس متعلق به سگهاى منطقه كرمـان، يسس از }
\end{aligned}
$$

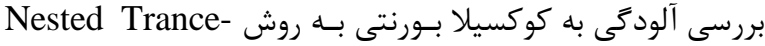

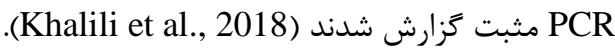

$$
\begin{aligned}
& \text { مطالعه حاضر اولين ززارش از زونههاى كنه سخت در بزهاى } \\
& \text { منطقه مشكين شهر و همجنين وضعيت آلودگى كنهها به } \\
& \text { كوكسيلا بورنتى در شمال غرب ايران است. با توجه به زئونوز }
\end{aligned}
$$

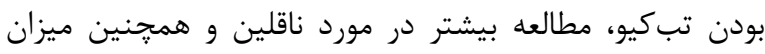

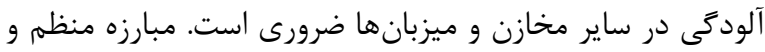

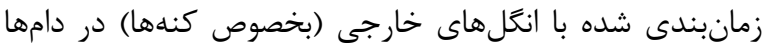

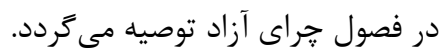

$$
\begin{aligned}
& \text { سياسگزן ارى } \\
& \text { نويسند } \\
& \text { نمونهاى اين تحقيق تشكر و قدردانى مى كنند. }
\end{aligned}
$$


Nourollahi-Fard, S.R. \& Khalili, M. 2011. PCRdetection of Coxiella burnetii in ticks collected from sheep and goats in southeast Iran. Iranian Journal of Arthropod-Borne Diseases 5: 1-6.

Ogawa M, Setiyono A, Sato K, Cai Y, Shiga S, Kishimoto T. 2004. Evaluation of PCR and nested PCR assays currently used for detection of Coxiella burnetii in Japan. Southeast Asia Journal of Tropical Medicine and Public Health 35: 852-855.

Parisi, A., Fraccalvieri, R., Cafiero, M., Miccolupo, A., Padalino, I., Montagna, C., Capuano, F. \& Sottili, R. 2006. Diagnosis of Coxiella burnetii-related abortion in Italian domestic ruminants using single-tube nested PCR. Veterinary Microbiology 118: 101-106.

Psaroulaki, A., Ragiadakou, D., Kouris, G., Papadopoulos, B., Chaniotis, B. \& Tselentis, Y. 2006. Ticks, tick-borne Rickettsiae, and Coxiella burnetii in the Greek island of Cephalonia. Annals of the New York Academy of Sciences 1078: 389-399.

Raoult, D., Marrie, T.J. \& Mege, J.L. 2005. Natural history and pathophysiology of Q fever. Lancet Infection Disease 5: 219-226.

Rasouli, S., Sadagian, M., Jafari, K., Valizadeh, E. \& Mojarad, M. 2010. Study on caprine hard tick fauna and seasonal variations of tick population in West Azarbaijan province. Veterinary Journal of Islamic Azad University of Tabriz 3: 667-671.

Sajid, M.S., Iqbal, Z., Khan, M.N., Muhammad, G. \& Khan, M.K. 2009. Prevalence and associated risk factors for bovine tick infestation in two districts of lower Punjab, Pakistan. Preventive Veterinary Medicine 92: 386-391.

Salimabadi, Y., Telmadarraiy, Z., Vatandoost, H., Chinikar, S., Oshaghi, M.A., Moradi, M., Mirabzadeh- Ardakan, E., Hekmat, S. \& Nasiri, A. 2010. Hard ticks on domestic ruminants and their seasonal population dynamics in Yazd Province, Iran. Iranian Journal of Arthropod-Borne Diseases 4: 6671.

Socolovschi, C., Reynaud, P., Kernif, T., Raoult, D. \& Parola, P. 2012. Rickettsiae of spotted fever group, Borrelia valaisiana, and Coxiella burnetii in ticks on passerine birds and mammals from the Camargue in the south of France. Tick and Tick-borne Diseases 3: $355-360$
Sohrabi, S., Yakhchali, M. \& Ghashghai, O. 2013. Hard ticks (Acarina: Ixodidae) diversity in the natural habitat of Iranian domestic ruminants: a provincial study in Kermanshah. Journal of Veterinary Research 68: 39-46.

Špitalská, E. \& Kocianova, E. 2003. Detection of Coxiella burnetii in ticks collected in Slovakia and Hungary. Europe Journal of Epidemiology 18: 263-266.

Vaidya, V.M., Malik, S., Simranpreet, K., Kumar, S. \& Barbuddh, S. 2008. Comparison of PCR, Immunoflourescence assay and pathogen isolation for diagnosis of $\mathrm{Q}$ fever in humans with spontaneous abortions. Journal of Clinical Microbiology 46: 20382044.

Vilcins, I.M., Old, J.M. \& Deane, E.M. 2005. The impact of ticks and tick-borne diseases on native animal species in Australia. Australian Journal of Microbiology 26: 76-78.

Walker, A., Bouattour, A. \& Camicas, J. 2003. Ticks of domestic animals in Africa: a guide to identification of species. $1^{\text {st }}$ ed. Scotland, (UK). Bioscience Report 1: 149-209.

Werede, H. \& Afera, B. 2014. Prevalence of ixodid ticks on bovine of Werieleke Wereda, Tigray. Acta Parasitologica 5: 146-150.

Yakhchali, M. \& Azizi, C. 2007. A study on ixodid tick infestation of cattle, sheep and goats in Bukan suburb, Iran. Iranian Journal of Veterinary Medicine 3: 100-104.

Yakhchali, M. \& Hajihasanzadehzarza, S.H. 2004. Study on some ecological aspects and prevalence of different species of hard ticks (Acarina: Ixodidae) on cattle, buffalo and sheep in Oshnavieh suburb. Pajuhesh \& Sazandegi 63: 31-35.

Yakhchali, M. \& Hosseine, A. 2006. Prevalence and ectoparasites fauna of sheep and goats flocks in Urmia suburb, Iran. Veterinary Arhive 76: 441-450.

Yakhchali, M. \& Ranjbargarmabolia, B. 2008. A study on ixodid ticks' fauna in sheep and goats of Salehabad in Torbatjam, Iran. Pajuhesh \& Sazandegi 80: 27-32.

Zabihi, R., Majidzadeh, K., Mohseni, M. \& Soleimani, M. 2014. Review on the Laboratory diagnosis of QFever. Journal of Army University of Medical Science 11: 383-388.

How to cite this article:

Esmaeilnejad, B., Gharekhani, J., Samiei, A. \& Rezaei, H. 2020. Molecular detection of Coxiella burnetii in ticks isolated from goats of Meshkin-Shahr County, Ardabil province. Nova Bioloica Reperta 7: 315-321. (In Persian).

اسمعيلنزاد، ب.، قرهخانى، ج.، سميعى، ا. و رضايى، ه.

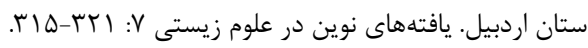

